\title{
Utility of Phosphorus Enhancers and Strip-Tillage for Corn Production
}

\author{
Christopher J. Dudenhoeffer ${ }^{1}$, Kelly A. Nelson ${ }^{1}$, Peter P. Motavalli ${ }^{2}$, Bruce Burdick ${ }^{3}$, \\ David Dunn ${ }^{4} \&$ Keith W. Goyne ${ }^{2}$ \\ ${ }^{1}$ Division of Plant Sciences, University of Missouri, Novelty, MO, USA \\ ${ }^{2}$ Department of Soil, Environmental and Atmospheric Sciences, University of Missouri, Columbia, MO, USA \\ ${ }^{3}$ Research Associate and Superintendent, Hundley-Whaley Center, University of Missouri, Albany, MO, USA \\ ${ }^{4}$ Division of Plant Sciences, University of Missouri, Portageville, MO, USA \\ Correspondence: Kelly Nelson, Division of Plant Sciences, University of Missouri, Novelty, MO, USA. Tel: \\ 1-660-739-4410. E-mail: nelsonke@missouri.edu
}

Received: November 26, 2012 Accepted: December 5, 2012 Online Published: January 15, 2013

doi:10.5539/jas.v5n2p37 URL: http://dx.doi.org/10.5539/jas.v5n2p37

\begin{abstract}
Farmers are seeking ways to diminish phosphorus (P) fertilizer rates and increase plant $\mathrm{P}$ uptake by means of enhanced efficiency $\mathrm{P}$ treatments. The objectives of this study were to determine the effects of tillage/fertilizer placement [no-till-surface broadcast (NT-BC) or strip-till-deep band (ST-DB)], monoammonium phosphate (MAP) rate $\left(0,56\right.$, and $\left.112 \mathrm{~kg} \mathrm{ha}^{-1}\right)$, and the presence or absence of enhanced phosphorus efficiency products (Avail $^{\circledR}$ and $\mathrm{P}_{2} \mathrm{O}_{5}-\mathrm{Max}^{\circledR}$ ) on corn (Zea mays L.) production. The field study was conducted in 2010 and 2011 at Novelty and Albany, MO. The two P enhancers had no effect on plant population, silage dry weights, grain moisture, yield, grain protein, grain starch, plant nitrogen $(\mathrm{N})$, potassium $(\mathrm{K})$ uptake, or apparent $\mathrm{P}$ recovery efficiency (APRE) at either location $(P>0.10)$. In the NT-BC and ST-DB treatments, the addition of Avail ${ }^{\circledR}$ or $\mathrm{P}_{2} \mathrm{O}_{5}-\mathrm{Max}^{\circledR}$ did not increase plant $\mathrm{P}$ uptake over the non-treated controls. ST-DB increased plant populations 3,500 to 15,500 plants $^{-1} \mathrm{a}^{-1}$ compared to NT-BC. At Novelty, yields increased $1.57 \mathrm{Mg}^{-1}$ with use of ST-DB over NT-BC, but at Albany yields were affected by tillage/fertilizer placement and MAP rate. Corn grain yields with MAP at $0 \mathrm{~kg} \mathrm{ha}^{-1}$ were 0.30 to $0.36 \mathrm{Mg} \mathrm{ha}^{-1}$ more than MAP at 56 or $112 \mathrm{~kg} \mathrm{P}_{2} \mathrm{O}_{5}$ ha $^{-1}$, which was probably due to the added ammonium nitrate used to balance the N contribution in MAP. Strip-till is a viable option to increase corn populations and yields on poorly drained soils, but $\mathrm{P}$ enhancers are not recommended for similar soil types.
\end{abstract}

Keywords: enhanced efficiency fertilizer products, fertilizer placement, monoammonium phosphate, no-till, phosphorus recovery

\section{Introduction}

Phosphorus (P) is a vital plant macronutrient. It serves as an important structural element in nucleic acids (RNA and DNA), as an energy transfer element (ATP), and it has a critical role in cellular regulation and carbon partitioning. Phosphorus fertilizers are used in large quantities in agriculture. The U. S. used $2.0 \times 10^{8} \mathrm{Mg}$ of $\mathrm{P}_{2} \mathrm{O}_{5}$ from 1960 to 2010 (USDA, 2012). In Missouri alone, $\mathrm{P}$ fertilizer use for corn production has increased from $4.4 \times 10^{4} \mathrm{Mg} \mathrm{P}_{2} \mathrm{O}_{5}$ in 1990 to $8 \times 10^{4} \mathrm{Mg} \mathrm{P}_{2} \mathrm{O}_{5}$ in 2010 (USDA, 2011).

In response to high fertilizer expenses, farmers seek ways to diminish $\mathrm{P}$ fertilizer application rates and use of $\mathrm{P}$ enhancers to increase P use efficiency. The manufacturers of Avail ${ }^{\mathbb{B}}$ (Specialty Fertilizer Products, Leawood, KS) and $\mathrm{P}_{2} \mathrm{O}_{5}-\mathrm{Max}^{\circledR}$ (P-Max, Rosen's Inc., Fairmont, MN) promoted these products as enhancing the efficiency of P-based fertilizers. Avail ${ }^{\circledR}$ is a $\mathrm{P}$ enhancer for liquid $\mathrm{P}$ fertilizers and granular phosphate fertilizers, including DAP and MAP. Avail ${ }^{\circledR}$ was intended to reduce the effect of cations (i.e., $\mathrm{Ca}, \mathrm{Fe}, \mathrm{Mn}$, and $\mathrm{Al}$ ) surrounding fertilizer granules on soil $\mathrm{P}$ sorption and plant $\mathrm{P}$ uptake. The maleic-itaconic copolymer in the product binds with these cations in soil solution to prevent $\mathrm{P}$ precipitation (SFP, 2009). $\mathrm{P}_{2} \mathrm{O}_{5}-\mathrm{Max}^{\circledR}$, containing the active ingredient poly-amino acid (L-aspartic acid)-sodium salt, reportedly increases $\mathrm{P}$ uptake and results in better nutrient absorption by improving root surface area (Rosen's Inc, 2012). However, mechanisms associated with product function are less clear. 
Little published research has investigated plant growth and yields in the presence of Avail ${ }^{\circledR}$. A study conducted in Kansas evaluated Avail ${ }^{\circledR}$ effectiveness in 2008 and 2009 at five locations under corn and wheat cropping systems (Ward, 2010). Plant biomass, grain yields, or P uptake for corn and wheat were not significantly affected by Avail ${ }^{\circledR}$. Two trials in Canada evaluated three rates of seed-placed MAP $\left(6.5,13,19.5 \mathrm{~kg} \mathrm{P} \mathrm{ha}^{-1}\right)$ and a nonfertilized control with or without Avail ${ }^{\circledR}$ (Karamanos \& Puurveen, 2011). For both wheat yield and P uptake, there was neither a significant effect of treating MAP with Avail ${ }^{\mathbb{B}}$, nor a significant interaction between Avail ${ }^{\mathbb{B}}$ treatment and rate of $\mathrm{P}$.

When evaluating fertilizer effectiveness, placement may increase efficiency. Erosion transports less P offsite when it is placed deep or incorporated as compared to broadcast surface application. Deep placement increased plant growth and yields compared to broadcast surface application (Hairston et al., 1990; Malhi et al., 2001). Malhi et al. (2001) compared the effectiveness of broadcast to deep banding of annual and one-time P fertilizer applications on alfalfa (Medicago sativa L.). Banding increased dry matter yield (DMY) between 742 to $954 \mathrm{~kg}$ $\mathrm{ha}^{-1}$ and protein yield between 173 to $205 \mathrm{~kg} \mathrm{ha}^{-1}$ compared to broadcast application. Banding resulted in greater P-use efficiency of applied P (58 kg DMY kg $\left.{ }^{-1} \mathrm{P} \mathrm{ha}^{-1}\right)$ compared to broadcast for an annual application and for the one-time application ( $47 \mathrm{~kg} \mathrm{DMY} \mathrm{kg}^{-1} \mathrm{P} \mathrm{ha}^{-1}$ ). Similarly, recovery of fertilizer $\mathrm{P}$ with deep banding was $16 \%$ greater for an annual application and $12 \%$ greater for the one-time application compared to broadcast application. However, the response of P fertilizers to deep placement has been inconsistent (Patrick et al., 1959; Mallarino et al., 1999; Borges \& Mallarino, 2001). Weather conditions also can affect plant response to deep P placement. Robertson et al. (1958) found significant corn yield increases with deep placement when residual P was present in the surface soil and total rainfall was adequate for plant growth with dry periods during early growth. However, deep placement did not affect yields when soil contained adequate residual $\mathrm{P}$ and rainfall was above average and well distributed throughout the growing season.

Conservation tillage practices, such as NT, have reduced erosion and lowered production costs. Minimal soil disturbance associated with no-till increased soil fertility, structure, and reduced potential for soil erosion (Triplett \& Dick, 2008). Adequate soil drainage and previous crop characteristics helped determine yield response to NT systems (Dick \& Van Doren, 1985; Guy \& Oplinger, 1989). Well-drained soils, crop rotation, and more southern latitudes generally benefited from NT during soybean production compared to poorly drained soils, continuous corn cropping systems, and northern latitudes (Griffith \& Wollenhaupt, 1994). On a Houston Black clay soil (fine, smectitic, thermic Udic Haplusterts), NT produced higher corn yields than a chisel tillage system without beds and a chisel tillage system with raised wide beds (Torbert et al., 2001).

No-till has reduced yields in some instances compared to conventional tillage (CT) due to lower soil temperatures and higher soil moisture early in the growing season, which reduced seedling emergence and slowed early growth (Burrows \& Larson, 1962; Fortin \& Pierce, 1990; Vyn \& Raimbult, 1993; Uri, 2000). No-till soils generally have greater bulk densities and penetrometer resistance (Bauder et al., 1981; Hill, 1990; Pierce et al., 1992) which can restrict root growth and affect fertilizer uptake. Studies have shown that NT corn yields were reduced by as much as 35\% compared with CT for moderately well to poorly drained soils (Erbach et al., 1992; Hussain et al., 1999). Halvorson et al. (2006) found CT produced 16\% higher yields than NT in Colorado. Lower grain yields associated with NT resulted from slow early-spring growth and delayed tasseling compared with the CT system as a result of cooler spring soil temperatures in NT. Cooler soil temperatures were due to greater residue cover on the soil surface in NT (89\%) than in CT (14\%). Howard et al. (2002) studied yield response between disk-till and NT in Tennessee. Disk-till yields were 0.59 to $1.34 \mathrm{Mg} \mathrm{ha}^{-1}$ greater than NT in 5 of the 11 site-years. However, P yield response was greater with NT production. No-till with P fertilizer application of $20 \mathrm{~kg} \mathrm{ha}^{-1}$ increased yields $0.62 \mathrm{Mg} \mathrm{ha}^{-1}$, while disk-till yields increased $0.44 \mathrm{Mg} \mathrm{ha}^{-1}$ with $\mathrm{P}$ application at $39 \mathrm{~kg} \mathrm{P} \mathrm{ha}^{-1}$.

Strip-till is another conservation tillage practice that aims to combine the yield benefits of tillage with the environmental improvements of NT. The practice consist of an implement tilling a narrow band, generally 15 to $20 \mathrm{~cm}$ wide and 15 to $20 \mathrm{~cm}$ deep, while leaving the rest of the soil undisturbed. In poorly drained soils, strip-till increased soil moisture evaporation, increased soil temperature (Bolton \& Booster, 1981) and decreased soil bulk densities in the row compared with NT (Drury et al., 2003; Overstreet \& Hoyt, 2008), which improves seedbed environment. Strip-till has been shown to increase corn yields compared to NT (Vetsch et al., 2007) and was equal to CT (Griffith et al., 1973; Randall et al., 2001). On continuous corn production in Minnesota, Vetsch and Randall (2002) observed a $0.4 \mathrm{Mg} \mathrm{ha}^{-1}$ increase with ST compared to NT. 
However, other research found limited yield differences between NT and ST (Mallarino et al., 1999; Al-Kaisi \& Lichet, 2004; Al-Kaisi \& Kwaw-Mensah, 2007; Archer \& Reicosky, 2009). In Iowa, Licht and Al-Kaisi (2005a) found that ST had no effect on $\mathrm{N}$ uptake, dry matter production, and corn grain yields compared to chisel plow and NT. Vetsch and Randall (2004) showed that CT increased corn grain yields $0.3 \mathrm{Mg}^{-1}$ over ST and $0.5 \mathrm{Mg}$ $\mathrm{ha}^{-1}$ over NT. However, silage yields were $0.8 \mathrm{Mg} \mathrm{ha}^{-1}$ greater for ST and $0.9 \mathrm{Mg} \mathrm{ha}^{-1}$ greater for CT compared to NT. Nitrogen uptake was also greater for ST $\left(193 \mathrm{~kg} \mathrm{ha}^{-1}\right)$ and CT $\left(198 \mathrm{~kg} \mathrm{ha}^{-1}\right)$ compared with NT $(181 \mathrm{~kg}$ $\mathrm{ha}^{-1}$ ). Perez-Bidegain et al. (2007) evaluated different tillage effects on corn and soybean yields in Iowa. No differences were observed for soybean yields between tillage systems, while corn planted with disk-chisel tillage yielded $0.8 \mathrm{Mg} \mathrm{ha}^{-1}$ more than the mean yield of ST and NT.

Increased fertilizer expenses, challenges of corn production with NT, and innovative P enhancer products available on the market prompted this research investigating techniques to enhance $\mathrm{P}$ fertilizer efficiency. The objective of this study was to assess the effect of tillage/fertilizer placement, $\mathrm{P}$ rate, and two $\mathrm{P}$ enhancer products on corn production, grain quality, $\mathrm{P}$ uptake, and apparent $\mathrm{P}$ recovery efficiency.

\section{Materials and Methods}

Field research was conducted in 2010 and 2011 at the Greenley Memorial Research Center $\left(40^{\circ} 01^{\prime} \mathrm{N}, 92^{\circ} 11^{\prime} \mathrm{W}\right)$ near Novelty, Mo., on a Kilwinning silt loam (fine, smectitic, mesis, Vertic Epiaqualfs) and at the Hundley-Whaley Center $\left(40^{\circ} 14^{\prime} \mathrm{N}, 94^{\circ} 20^{\prime} \mathrm{W}\right)$ near Albany, Mo., on a Bremer silty clay loam (fine, smectitic, mesic, Typic Argiaquolls). Each site was arranged as a factorial randomized complete block design with four replications. Corn was planted following soybean. Pre-treatment soil conditions were evaluated from $15-\mathrm{cm}$ depth samples randomly collected from each replication and analyzed by the University of Missouri Soil and Plant Testing Laboratory using standard methods (Nathan et al., 2006) including soil $\mathrm{pH}\left(0.01 \mathrm{M} \mathrm{CaCl}_{2}\right)$, Bray-1 $\mathrm{P}$, exchangeable potassium, calcium, magnesium $\left(1 \mathrm{M} \mathrm{NH}_{4} \mathrm{OA}_{\mathrm{C}}\right.$ ), zinc (DTPA extraction), soil organic matter (loss-on-ignition), neutralizable acidity (Woodruff buffer), and effective cation exchange capacity (Table 1).

Table 1. Selected initial soil properties for the P placement, rate, and enhancer experiments at Novelty and Albany in 2010 and 2011

\begin{tabular}{|c|c|c|c|c|}
\hline \multirow{2}{*}{ Soil properties } & \multicolumn{2}{|c|}{ Novelty } & \multicolumn{2}{|c|}{ Albany } \\
\hline & 2010 & 2011 & 2010 & 2011 \\
\hline $\mathrm{pH}_{\mathrm{s}}\left(0.01 \mathrm{M} \mathrm{CaCl}_{2}\right)$ & $6.8 \pm 0.3^{\top}$ & $6.6 \pm 0.2$ & $6.4 \pm 0.4$ & $6.0 \pm 0.3$ \\
\hline Bray-1 P $\left(\mathrm{kg} \mathrm{ha}^{-1}\right)$ & $50 \pm 26$ & $27 \pm 8$ & $90 \pm 48$ & $90 \pm 11$ \\
\hline \multicolumn{5}{|l|}{ Exchangeable $\left(1 \mathrm{M} \mathrm{NH}_{4} \mathrm{OA}_{\mathrm{C}}\right)$} \\
\hline Potassium $\left(\mathrm{kg} \mathrm{ha}^{-1}\right)$ & $275 \pm 41$ & $158 \pm 25$ & $293 \pm 25$ & $315 \pm 44$ \\
\hline Calcium $\left(\mathrm{kg} \mathrm{ha}^{-1}\right)$ & $6039 \pm 528$ & $5585 \pm 242$ & $6466 \pm 417$ & $6513 \pm 692$ \\
\hline Magnesium $\left(\mathrm{kg} \mathrm{ha}^{-1}\right)$ & $470 \pm 83$ & $502 \pm 54$ & $713 \pm 200$ & $779 \pm 109$ \\
\hline Zinc $\left(\mathrm{mg} \mathrm{kg}{ }^{-1}\right)$ (DTPA Extraction) & $0.75 \pm 0.24$ & $0.35 \pm 0.13$ & $0.80 \pm 0.24$ & $0.88 \pm 0.17$ \\
\hline Soil organic matter $\left(\mathrm{g} \mathrm{kg}^{-1}\right)$ & $24 \pm 8$ & $26 \pm 1$ & $25 \pm 1$ & $27 \pm 7$ \\
\hline Neutralizable acidity $\left(\mathrm{cmol}_{\mathrm{c}} \mathrm{kg}^{-1}\right)$ & $0.25 \pm 0.5$ & $0.75 \pm 0.29$ & $1.25 \pm 1.19$ & $2 \pm 0.82$ \\
\hline Cation exchange capacity $\left(\mathrm{cmol}_{\mathrm{c}} \mathrm{kg}^{-1}\right)$ & $16 \pm 1$ & $15 \pm 1$ & $19 \pm 2$ & $20 \pm 3$ \\
\hline
\end{tabular}

Treatments included a three-factor arrangement of fertilizer placement (NT/surface broadcast or strip-till/deep band), monoammonium phosphate (MAP) rate $\left(0,56 \mathrm{~kg} \mathrm{P}_{2} \mathrm{O}_{5} \mathrm{ha}^{-1}, 112 \mathrm{~kg} \mathrm{P}_{2} \mathrm{O}_{5} \mathrm{ha}^{-1}\right)$, and the presence or absence of two enhanced phosphorus efficiency products [non-treated control, Avail ${ }^{\mathbb{R}}$ at $2.1 \mathrm{~L} \mathrm{Mg}^{-1}$ of fertilizer, and $\mathrm{P}_{2} \mathrm{O}_{5} \mathrm{Max}^{\circledR}$ at $4.2 \mathrm{~L} \mathrm{Mg}^{-1}$ of fertilizer]. At Novelty, $\mathrm{P}$ fertilizer treatments were deep banded using a Yetter ${ }^{\circledR}$ 2984 strip-till system equipped with high residue Maverick ${ }^{\circledR}$ units (Yetter Manufacturing, Inc., Colchester, IL), a rolling basket, and dry fertilizer application tubes. At Albany, P fertilizer treatments also were deep banded using a Yetter ${ }^{\mathbb{B}} 2984$ strip-till system equipped with residue manager wheels, B-33 mole knife, and opposing closing wheel disks. A Gandy Orbit Air ${ }^{\mathbb{B}}$ (Gandy Company, Owatonna, MN) dry fertilizer applicator metered and delivered fertilizer behind the applicator knife in the strip till system. Phosphorus was applied with a hand spreader in the NT surface broadcast treatment. Ammonium nitrate fertilizer was broadcast-applied for the appropriate treatments to balance the N contribution of MAP as the rate was reduced. The planter was equipped with Shark-tooth ${ }^{\circledR}$ (Yetter Manufacturing, Inc., Colchester, IL) residue cleaners used in tandem with a NT 
coulter. The residue cleaners performed well in heavy residue of the NT plots and provided a smooth seedbed above strip-tilled plots. The row spacing was $0.76-\mathrm{m}$. Management information is presented in Table 2.

Table 2. Field and management information for P placement, rate, and enhancer experiments at Novelty and Albany in 2010 and 2011

\begin{tabular}{|c|c|c|c|c|}
\hline \multirow{2}{*}{$\begin{array}{l}\text { Management } \\
\text { information }\end{array}$} & \multicolumn{2}{|l|}{ Novelty } & \multicolumn{2}{|l|}{ Albany } \\
\hline & 2010 & 2011 & 2010 & 2011 \\
\hline Plot size & 3 by $23 \mathrm{~m}$ & 3 by $23 \mathrm{~m}$ & 4.6 by $23 \mathrm{~m}$ & 4.6 by $23 \mathrm{~m}$ \\
\hline Hybrid & DK 62-54 VT3 & DK 62-54 VT3 & DK 63-84 VT3 & DK 63-84 VT3 \\
\hline Planting date & 14 Apr. & 31 Mar. & 30 May & 13 Apr. \\
\hline Seeding rate & 74,100 seeds $\mathrm{ha}^{-1}$ & 74,100 seeds $\mathrm{ha}^{-1}$ & 74,100 seeds $\mathrm{ha}^{-1}$ & 72,900 seeds $\mathrm{ha}^{-1}$ \\
\hline $\begin{array}{l}\text { Tissue harvest } \\
\text { date }\end{array}$ & $7 \mathrm{Sep}$. & 25 Aug. & 9 Sep. & 26 Aug. \\
\hline Harvest date & 30 Oct. & 8 Sep. & 15 Oct. & 27 Sep. \\
\hline $\begin{array}{l}\mathrm{P} \text { fertilizer } \\
\text { application date }\end{array}$ & 13 Apr. & 30 Mar. & 15 Apr. & 15 Nov. \\
\hline $\begin{array}{l}\text { Additional } \\
\text { fertilizer (date, } \\
\text { source, \& rate) }\end{array}$ & $\begin{array}{l}6 \text { May, Urea }\left(202 \mathrm{~kg} \mathrm{~N} \mathrm{ha}^{-1}\right) \\
+\operatorname{NBPT}\left(4 \mathrm{~L} \mathrm{Mg}^{-1}\right)\end{array}$ & $\begin{array}{c}31 \text { Oct. } 2010, \\
\text { Anhydrous } \\
\text { ammonia }(179 \mathrm{~kg} \mathrm{~N} \\
\left.\mathrm{ha}^{-1}\right)+ \text { Nitrapyrin } \\
\left(0.56 \mathrm{~kg} \mathrm{a.i.} \mathrm{ha}^{-1}\right)\end{array}$ & $\begin{array}{l}19 \text { Apr., Urea }\left(168 \mathrm{~kg} \mathrm{~N} \mathrm{ha}^{-1}\right) \\
\quad+\operatorname{NBPT}\left(4 \mathrm{~L} \mathrm{Mg}^{-1}\right)\end{array}$ & $\begin{array}{c}14 \text { Apr., Urea (168 kg } \\
\left.\text { ha }^{-1}\right)\end{array}$ \\
\hline
\end{tabular}

Weed

management ${ }^{*}$

\begin{tabular}{|c|c|c|c|c|}
\hline Burndown & NA & $\begin{array}{l}\text { 11 Apr., Glyphosate } \\
\left(1.06 \mathrm{~kg} \text { a.i. } \mathrm{ha}^{-1}\right)+ \\
\text { DS }\left(0.36 \mathrm{~kg} \mathrm{a}^{\mathrm{i}} .\right. \\
\left.\mathrm{ha}^{-1}\right)+ \text { DAS }(512 \\
\left.\mathrm{mL} \mathrm{ha}^{-1}\right)\end{array}$ & NA & NA \\
\hline Preemergence & $\begin{array}{c}16 \text { Apr., S-metolachlor }(2.25 \\
\left.\text { kg a.i. } \mathrm{ha}^{-1}\right)+ \text { Atrazine }(0.84 \\
\left.\text { kg a.i. } \mathrm{ha}^{-1}\right)+ \text { Mesotrione } \\
\left(0.23 \mathrm{~kg} \mathrm{a}^{-1 .} \mathrm{ha}^{-1}\right)+\text { DSD } \\
\left(0.56 \mathrm{~kg} \mathrm{a.i.} \mathrm{ha}^{-1}\right)\end{array}$ & $\begin{array}{l}13 \text { Apr., Acetochlor } \\
\left(2.35 \mathrm{~kg} \text { a.i. } \mathrm{ha}^{-1}\right)+ \\
\text { Atrazine }(1.77 \mathrm{~kg} \\
\left.\text { a.i. } \mathrm{ha}^{-1}\right)\end{array}$ & $\begin{array}{l}15 \text { Apr., S-metolachlor }(2.4 \\
\left.\text { kg a.i. } \mathrm{ha}^{-1}\right)+ \text { Atrazine }(0.9 \\
\left.\mathrm{kg} \text { a.i. } \mathrm{ha}^{-1}\right)+ \text { Mesotrione } \\
\left(0.24 \mathrm{~kg} \mathrm{a.i.}^{-1}\right) ; 30 \text { May, } \\
\text { Isoxaflutole }\left(0.14 \mathrm{~kg}^{-1 . i .} \mathrm{ha}^{-1}\right)\end{array}$ & $\begin{array}{c}\text { 16 Apr., S-metolachlor } \\
\left(2.4 \mathrm{~kg} \text { a.i. } \mathrm{ha}^{-1}\right)+ \\
\text { Atrazine }(0.9 \mathrm{~kg} \text { a.i. } \\
\left.\mathrm{ha}^{-1}\right)+ \text { Mesotrione }(0.24 \\
\left.\mathrm{kg} \text { a.i. } \mathrm{ha}^{-1}\right)\end{array}$ \\
\hline Postemergence & $\begin{array}{c}22 \text { June, Glyphosate }(1.45 \\
\left.\text { kg a.i. ha }{ }^{-1}\right)+ \text { DAS }(0.04 \mathrm{~kg} \\
\left.\text { a.i. } \mathrm{ha}^{-1}\right)\end{array}$ & NA & $\begin{array}{l}21 \text { June, Glyphosate } \\
\left(1.16 \mathrm{~kg} \text { a.i. } \mathrm{ha}^{-1}\right)\end{array}$ & $\begin{array}{l}7 \text { June, Glyphosate } \\
\left(1.16 \mathrm{~kg} \text { a.i. } \mathrm{ha}^{-1}\right)\end{array}$ \\
\hline
\end{tabular}

${ }^{\dagger}$ Abbreviations: DAS, Diammonium sulfate; DS, Dimethylamine salt; DSD, Dimethylamine salt of dicamba; NA, None applied.

*Acetochlor

(2-chloro-4-(ethylamino)-6-(isopropylamino)-s-triazine); diammonium sulfate ((NH4)2SO4); dimethylamine salt (2,4-Dichlorophenoxyacetic acid); dimethylamine salt of dicamba (3,6-dichloro-0-anisic acid); glyphosate (N-(phosphonomethyl)glycine); isoxaflutole (5-cyclopropyl-4-(2-methylsulfonyl-4-trifluoromethylbenzoyl) isoxazole); mesotrione (2-[4-(Methylsulfonyl)-2-nitrobenzoyl]cyclohexane-1,3-dione); nitrapyrin (2-chloro-6-(trichloromethyl) pyridine); NBPT (N-(n-butyl) thiophosphoric triamide); S-metolachlor (2-chloro-N-(2-ethyl-6-methylphenyl)-N-[(1S)-2-methoxy-1-methylethyl]acctamide).

The two center rows of each plot were harvested with a plot combine (Wintersteiger Delta, Salt Lake City, UT) and were used to measure corn grain yield and moisture content. Grain starch, protein, and oil concentration (Foss Infratec, Eden Prairie, MN) were collected from each plot. Grain yields were adjusted to $155 \mathrm{~g} \mathrm{~kg}^{-1}$ moisture before analysis. At physiological maturity, $1.5 \mathrm{~m}$ of one row was harvested and used to measure corn silage yield expressed on a dry matter basis. The silage samples underwent a $\mathrm{H}_{2} \mathrm{SO}_{4}-\mathrm{H}_{2} \mathrm{O}_{2}$ digestion and were analyzed for total $\mathrm{N}$ (colorimetric Indophenols blue), P (colorimetric ammonium molybdate), and $\mathrm{K}$ (atomic absorption) concentration. Apparent P recovery efficiency (APRE) was calculated as [((kg P uptake ha ${ }^{-1}$ of treated - $\mathrm{kg}$ P uptake ha ${ }^{-1}$ of control)/(kg fertilizer applied $\left.\left.\left.\mathrm{P} \mathrm{ha}^{-1}\right)\right)^{*} 100\right]$. All data were subjected to analysis of 
variance and means separation using Fisher's Protected LSD $(P=0.1)$. Data were combined over factors and locations when appropriate as indicated by the analysis of variance (data not presented). Plant population at Novelty and Albany, and grain oil concentration at Novelty were subjected to an $F$ Max test for homogeneity (Kuehl, 1994) and combined over site-years when variances were homogenous.

\section{Results and Discussions}

Precipitation at the study sites during the 2010 growing season was $265 \mathrm{~mm}$ at Albany and $523 \mathrm{~mm}$ at Novelty greater than during 2011 (Figure 1). At Novelty, the 2010 growing season received the greatest precipitation (1082 mm) of all four site years which was $362 \mathrm{~mm}$ greater than the average precipitation for the past decade (Nelson et al., 2010). Soil temperatures at a depth of $5.1 \mathrm{~cm}$ were similar for all locations within a particular year, but temperatures differed by year (Figure 2). No four-way interactions (year*P enhancer*placement*MAP rate) existed for the parameters evaluated; thus, main effects are reported and interactions presented when appropriate.
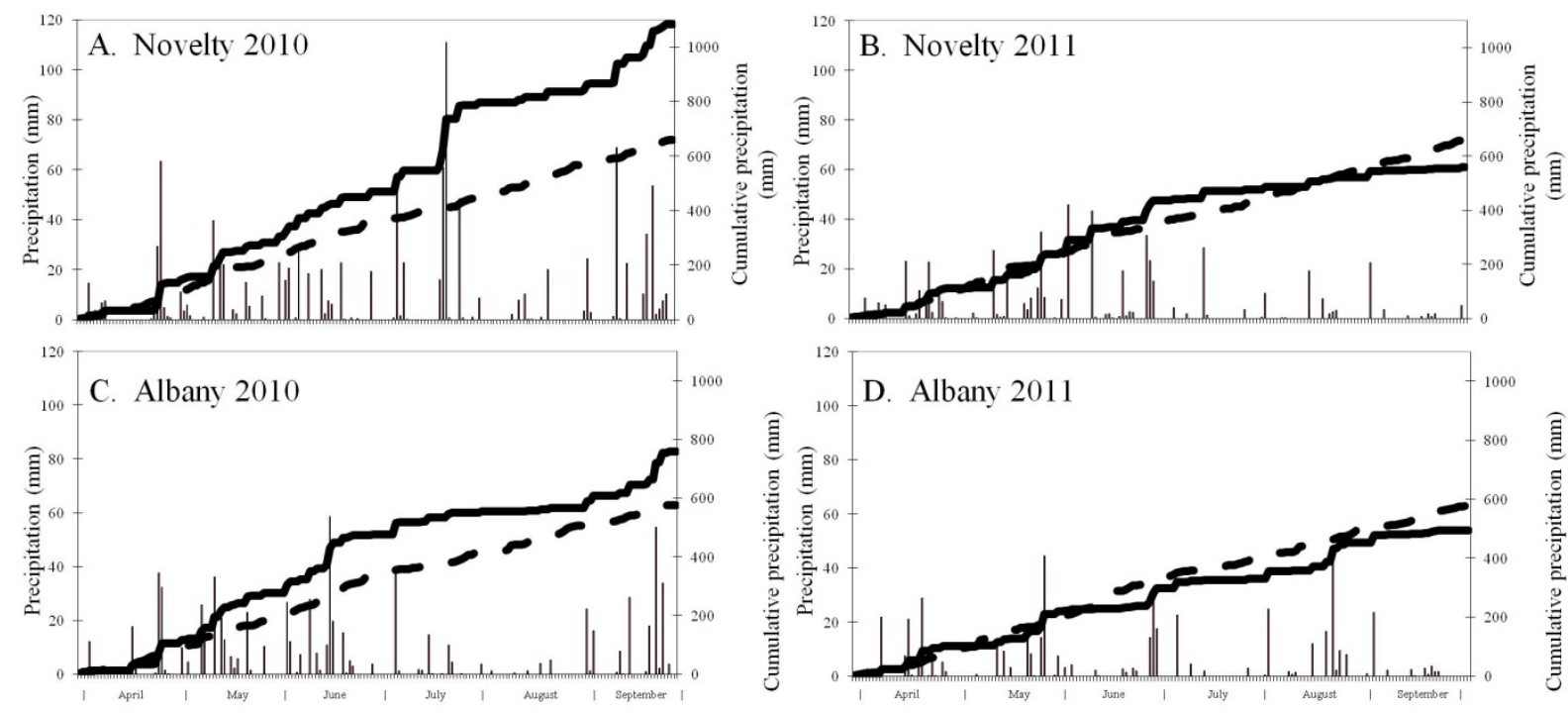

Figure 1. Daily (bars) and cumulative (solid line) precipitation from March through September of 2010 and 2011 at Novelty (A and B) and Albany (C and D). Dashed lines are the cumulative precipitation from the last decade for each location

\subsection{Phosphorus Enhancer}

Table 3. The effect of $\mathrm{P}$ enhancer on grain oil and $\mathrm{P}$ uptake

\begin{tabular}{|c|c|c|c|c|}
\hline \multirow{3}{*}{ P enhancer } & \multirow{2}{*}{\multicolumn{2}{|c|}{ Oil }} & \multicolumn{2}{|c|}{ P uptake } \\
\hline & & & \multicolumn{2}{|c|}{ Placement } \\
\hline & Novelty $^{\top}$ & Albany $^{\top}$ & NT/broadcast & ST/deep banding \\
\hline & \multicolumn{2}{|c|}{---------- $\mathrm{g} \mathrm{kg}^{-1}$---------- } & \multicolumn{2}{|c|}{------------------kg ha' ${ }^{-1}-----------------$} \\
\hline Non-treated & 36.8 & 37.7 & 39.6 & 38.1 \\
\hline Avail $^{(\mathbb{R})}$ & 36.4 & 36.9 & 38.0 & 39.8 \\
\hline $\mathrm{P}_{2} \mathrm{O}_{5}-\mathrm{Max}^{(\mathrm{B})}$ & 36.9 & 36.4 & 40.0 & 34.1 \\
\hline $\operatorname{LSD}(P=0.1)$ & $\mathrm{NS}^{+}$ & 0.7 & \multicolumn{2}{|c|}{-------------------4.1 ------------------- } \\
\hline P-value & 0.74 & 0.01 & \multicolumn{2}{|c|}{------------------0.09------------------ } \\
\hline
\end{tabular}

${ }^{\dagger}$ Data were combined over years (2010 and 2011).

${ }^{*}$ Data were combined over site-year and MAP (monoammonium phosphate) rate.

${ }^{\S} \mathrm{NS}=$ Not significant. 
The $\mathrm{P}$ enhancers did not affect plant population $(P=0.51)$, silage dry weights $(P=0.81)$, grain moisture $(P=0.54)$, yield $(P=0.83)$, grain protein $(P=0.74)$, grain starch $(P=0.63), \mathrm{N}$ uptake $(P=0.42), \mathrm{K}$ uptake $(P=0.82)$, or APRE $(P=0.32)$ during the four site-years (data not presented). At Albany, oil concentration in the non-treated control was $1.3 \mathrm{~g} \mathrm{~kg}^{-1}$ greater than $\mathrm{P}_{2} \mathrm{O}_{5}-\mathrm{Max}^{\circledR}$ (Table 3). In the NT-BC and ST-DB treatments, the addition of Avail ${ }^{\circledR}$ or $\mathrm{P}_{2} \mathrm{O}_{5}-\mathrm{Max}^{\circledR}$ did not increase P uptake over the non-treated controls. Avail ${ }^{\circledR}$ increased $\mathrm{P}$ uptake $5.7 \mathrm{~kg} \mathrm{ha}^{-1}$ over $\mathrm{P}_{2} \mathrm{O}_{5}-\mathrm{Max}^{\circledR}$ with ST-DB, and no differences were observed between products in the NT-BC treatment.

Phosphorus uptake increased $5.9 \mathrm{~kg} \mathrm{ha}^{-1}$ when $\mathrm{P}$ fertilizer was applied with $\mathrm{P}_{2} \mathrm{O}_{5}-\mathrm{Max}^{\circledR}$ and NT-BC instead of ST-DB. In Kansas, Ward (2010) found similar results with no significant effect of Avail ${ }^{\circledR}$ for corn or wheat biomass production, $\mathrm{P}$ uptake, or grain yields of either crop. Neither a significant effect of treating MAP with Avail $^{\circledR}$, nor a significant interaction between Avail ${ }^{\circledR}$ treatment and rate of $\mathrm{P}$ on the yield of wheat and $\mathrm{P}$ uptake was shown in Canada (Karamanos \& Puurveen, 2011).

\subsection{Phosphorus Placement}

Strip-till/deep band increased plant populations 15,500 plants $^{-1} \mathrm{a}^{-1}$ at Novelty and 3,500 plants ha ${ }^{-1}$ at Albany compared to NT-BC (Table 4). The claypan soil at Novelty has poorer internal drainage than the Bremer silt loam at Albany. With ST, an improved the seedbed environment likely caused the greater plant populations. In poorly drained soils, strip-till can increased soil moisture evaporation, increased soil temperature (Bolton \& Booster, 1981) and decreased soil bulk densities in the row compared with NT (Drury et al., 2003; Overstreet \& Hoyt, 2008), which improves seedbed environment. This was particularly important early in the growing season. Licht and Al-Kaisi (2005b) evaluated the effect of tillage on soil temperature. In the top $5 \mathrm{~cm}$, ST increased soil temperature $1.2^{\circ} \mathrm{C}$ to $1.4^{\circ} \mathrm{C}$ over NT. This caused the corn emergence rate index of ST to be slightly greater than NT throughout the four site years (Licht \& Al-Kaisi, 2005b). This effect could have been important in maintaining a good corn stand during April and early May when there was higher rainfall (Figure 1) and cooler soil temperatures (Figure 2).

Table 4. Phosphorus placement effect on plant population, grain moisture, yield, and apparent $\mathrm{P}$ recovery efficiency (APRE)

\begin{tabular}{|c|c|c|c|c|c|c|c|c|}
\hline \multirow[b]{4}{*}{ Placement } & & & & \multicolumn{4}{|c|}{ Grain yield } & \multirow[b]{4}{*}{ APRE } \\
\hline & \multirow{2}{*}{\multicolumn{2}{|c|}{$\begin{array}{c}\text { Plant } \\
\text { population }\end{array}$}} & \multirow{3}{*}{$\begin{array}{c}\text { Grain } \\
\text { moisture }\end{array}$} & \multirow[b]{3}{*}{ Novelty $^{\dagger}$} & \multirow{2}{*}{\multicolumn{3}{|c|}{$\frac{\text { Albany }^{\dagger}}{\text { MAP rate }\left(\mathrm{kg} \mathrm{P}_{2} \mathrm{O}_{5} \mathrm{ha}^{-1}\right)}$}} & \\
\hline & & & & & & & & \\
\hline & Novelty $^{\dagger}$ & Albany $^{\dagger}$ & & & 0 & 56 & 112 & \\
\hline & \multicolumn{2}{|c|}{----plants ha ${ }^{-1}-----$} & $\mathrm{g} \mathrm{kg}^{-1}$ & - --- & $---M$ & - & ---- & $\%$ \\
\hline NT/broadcast & 50,600 & 57,600 & 172.2 & 6.74 & 9.32 & 8.61 & 8.97 & 21.4 \\
\hline ST/deep banding & 66,100 & 61,100 & 168.9 & 8.31 & 8.77 & 8.90 & 9.05 & 0.7 \\
\hline $\operatorname{LSD}(P=0.1)$ & 2,600 & 2,900 & 2.4 & 0.36 & -----. & --0.47 & ------- & 11.5 \\
\hline
\end{tabular}

Data were combined over years (2010 and 2011).

${ }^{+} \mathrm{NT}=$ No-till; ST = Strip-till.
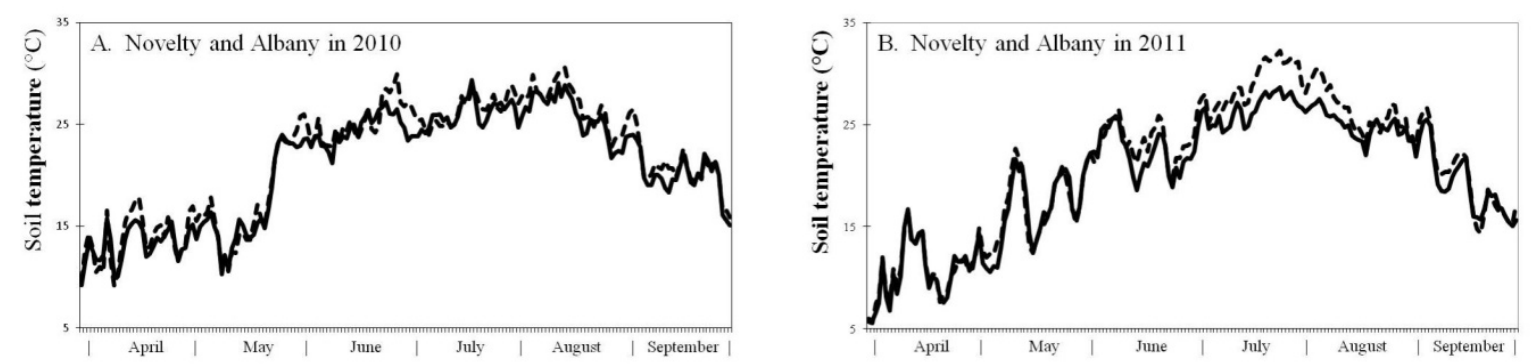

Figure 2. Average daily soil temperature at $51 \mathrm{~mm}$ depth from March through September in 2010 (A) and 2011 (B) at Novelty and Albany. The solid line is Novelty and the dashed line is Albany 
Tillage/fertilizer placement did not affect silage dry weights, N, or K uptake. However, grain moisture was $3.3 \mathrm{~g}$ $\mathrm{kg}^{-1}$ greater in NT-BC compared to ST-DB (Table 4). No-till/broadcast increased APRE 20.7\% over ST-DB. At Novelty, yields increased $1.57 \mathrm{Mg} \mathrm{ha}^{-1}$ with use of ST-DB over NT-BC, but yields at Albany were affected by tillage/fertilizer placement and MAP rate. When no MAP was added at Albany, NT-BC increased grain yields $0.55 \mathrm{Mg} \mathrm{ha}^{-1}$ over ST-DB. However, no difference was observed between NT-BC and ST-DB with MAP at 56 or $112 \mathrm{~kg} \mathrm{P}_{2} \mathrm{O}_{5} \mathrm{ha}^{-1}$. Fertilization with MAP at $0 \mathrm{~kg} \mathrm{P}_{2} \mathrm{O}_{5}$ ha $^{-1}$ yielded $0.71 \mathrm{Mg} \mathrm{ha}^{-1}$ more than MAP at $56 \mathrm{~kg} \mathrm{P}_{2} \mathrm{O}_{5}$ $\mathrm{ha}^{-1}$ under NT-BC, but no difference was observed with MAP at $112 \mathrm{~kg} \mathrm{P}_{2} \mathrm{O}_{5} \mathrm{ha}^{-1}$. This difference may be due to the addition of ammonium nitrate in the $0 \mathrm{~kg} \mathrm{P}_{2} \mathrm{O}_{5}$ ha $^{-1}$ control to balance the $\mathrm{N}$ contribution as the MAP rate increased. The soils in these experiments have a high potential for gaseous fertilizer $\mathrm{N}$ loss due to poor internal soil drainage and long periods of soil saturation, especially when urea is the $\mathrm{N}$ source (Nash et al., 2012). Urea was selected as the $\mathrm{N}$ source at three of the four site-years due the inability to apply anhydrous ammonia. The lack of response at Albany to the addition of MAP could also result from greater initial Bray-1 P compared to Novelty (Table 1). Strip-till has been shown to increase corn yields compared to NT (Vetsch et al., 2007), and had yields similar to CT in other research (Griffith et al., 1973; Randall et al., 2001). In Minnesota, Vetsch and Randall (2002) found in continuous corn that yield increased $0.4 \mathrm{Mg} \mathrm{ha}^{-1}$ with ST compared to NT. However, studies have shown yield differences between NT and ST ranging from none to limited (Mallarino et al., 1999; Al-Kaisi \& Lichet, 2004; Al-Kaisi \& Kwaw-Mensah, 2007; Archer \& Reicosky, 2009). In Iowa, Licht and Al-Kaisi (2005a) found that ST did not affect N uptake, dry matter production, or corn grain yields compared to chisel plow and NT. Also in Iowa, Perez-Bidegain et al. (2007) evaluated different tillage effects on corn and soybean yields. They observed no differences for soybean yields between tillage systems, while corn planted with disk-chisel tillage yielded $0.8 \mathrm{Mg} \mathrm{ha}^{-1}$ more than the mean yield of ST and NT.

Grain protein and starch concentrations exhibited an interaction between year and placement at Novelty, but not at Albany (Table 5). The NT-BC treatment had $14 \mathrm{~g} \mathrm{~kg}^{-1}$ greater protein concentration in 2010 than ST-DB and 6 $\mathrm{g} \mathrm{kg}^{-1}$ greater protein concentration in 2011. In 2010 and 2011, ST-DB increased starch concentration by 8 and 3 $\mathrm{g} \mathrm{kg}^{-1}$, respectively. Grain oil concentration was affected by location, placement, and MAP rate. At Novelty, NT-BC with MAP at $0 \mathrm{~kg} \mathrm{P}_{2} \mathrm{O}_{5} \mathrm{ha}^{-1}$ had at least $1.7 \mathrm{~g} \mathrm{~kg}^{-1}$ less oil concentration than any other placement-MAP rate combination. At Albany, ST-DB with MAP at $0 \mathrm{~kg} \mathrm{P}_{2} \mathrm{O}_{5} \mathrm{ha}^{-1}$ had a lower oil concentration than any other placement-MAP rate combination except for NT-BC MAP at $112 \mathrm{~kg} \mathrm{P}_{2} \mathrm{O}_{5} \mathrm{ha}^{-1}$. The effect of tillage on plant stand was less pronounced at Albany than at Novelty which may have affected grain quality.

Table 5. Placement effect on grain protein, starch, and oil. Data were combined over MAP rate and P stabilizer except for grain oil which was combined over site-year and P stabilizer

\begin{tabular}{|c|c|c|c|c|c|c|c|c|c|c|c|c|}
\hline \multirow[b]{4}{*}{ Placement } & \multirow{2}{*}{\multicolumn{3}{|c|}{ Protein }} & \multirow{2}{*}{\multicolumn{3}{|c|}{ Starch }} & \multicolumn{6}{|c|}{ Oil } \\
\hline & & & & & & & \multirow{2}{*}{\multicolumn{3}{|c|}{$\frac{\text { Novelty }^{\dagger}}{\text { MAP rate }\left(\mathrm{kg} \mathrm{P}_{2} \mathrm{O}_{5} \mathrm{ha}^{-1}\right)}$}} & \multicolumn{3}{|c|}{ Albany $^{\dagger}$} \\
\hline & \multicolumn{2}{|c|}{ Novelty } & \multirow[b]{2}{*}{ Albany $^{\dagger}$} & \multicolumn{2}{|c|}{ Novelty } & \multirow[b]{2}{*}{ Albany $^{\dagger}$} & & & & \multicolumn{3}{|c|}{ MAP rate $\left(\mathrm{kg} \mathrm{P}_{2} \mathrm{O}_{5} \mathrm{ha}^{-1}\right)$} \\
\hline & 2010 & 2011 & & 2010 & 2011 & & 0 & 56 & 112 & 0 & 56 & 112 \\
\hline & \multicolumn{3}{|c|}{ - $\mathrm{g} \mathrm{kg}^{-1}-$} & \multicolumn{3}{|c|}{ 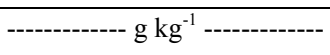 } & \multicolumn{3}{|c|}{----- g kg$^{-1}$------ } & \multicolumn{3}{|c|}{------ g kg$^{-1}$------- } \\
\hline NT/broadcast & 84 & 93 & 84 & 739 & 729 & 723 & 34.8 & 37.3 & 36.5 & 37.4 & 37.3 & 36.8 \\
\hline ST/deep banding & 70 & 87 & 85 & 747 & 732 & 722 & 37.5 & 37.5 & 36.7 & 36.0 & 37.6 & 37.0 \\
\hline $\operatorname{LSD}(P=0.1)$ & 2 & 2 & $\mathrm{NS}^{+}$ & 2 & 2 & NS & \multicolumn{3}{|c|}{----------1.4----------- } & \multicolumn{3}{|c|}{----------1.0---------- } \\
\hline
\end{tabular}

${ }^{\dagger}$ Data were combined over years (2010 and 2011).

${ }^{*} \mathrm{NS}=$ Not significant; NT = No-till; ST $=$ Strip-till.

\subsection{Monoammonium Phosphate Rate}

MAP rate did not affect plant population, silage dry weights, and grain moisture at all four site-years (data not presented). Corn grain yields with MAP at $0 \mathrm{~kg} \mathrm{ha}^{-1}$ were 0.30 to $0.36 \mathrm{Mg} \mathrm{ha}^{-1}$ more than MAP at 56 or $112 \mathrm{~kg} \mathrm{P}_{2} \mathrm{O}_{5}$ $\mathrm{ha}^{-1}$ (Table 6). This difference may be due to the addition of ammonium nitrate to the $0 \mathrm{~kg} \mathrm{P}_{2} \mathrm{O}_{5}$ ha $^{-1}$ control that was added to balance the $\mathrm{N}$ contribution as the MAP rate increased to $112 \mathrm{~kg} \mathrm{ha}^{-1}$. There were no significant differences in $\mathrm{N}$ uptake between MAP rates, but the MAP rate at $0 \mathrm{~kg} \mathrm{P}_{2} \mathrm{O}_{5} \mathrm{ha}^{-1}$ indicated greater $\mathrm{N}$ uptake than MAP at 56 or $112 \mathrm{~kg} \mathrm{P}_{2} \mathrm{O}_{5}$ ha $^{-1}$. The effectiveness of DAP as a dual source of $\mathrm{P}$ and $\mathrm{N}$ for corn was evaluated in a greenhouse study (Lu et al., 1987). On a calcareous clay soil, DAP was compared with urea plus single superphosphate (SSP) placed at different depths (deep banding, surface broadcast, and incorporation). Soil treated with DAP contained less Olsen P than soil treated with urea plus SSP regardless of fertilizer placement. Reduced P 
accessibility to the plant roots resulting in lower $P$ uptake and plant yield occurred with both fertilizers when they were surface broadcast compared to the incorporation treatments. When the fertilizers were incorporated or deep-placed, $\mathrm{N}$ uptake was as great with DAP as with urea plus SSP. However, surface application resulted in higher N uptake from urea plus SSP than DAP. Regardless of fertilizer placement, urea plus SSP produced greater plant yields than those obtained with DAP.

Table 6. Yield, $\mathrm{N}$ uptake, protein, and starch concentration as affected by MAP rate. Data were combined over site-year, location, placement, and P stabilizer except for grain protein and starch

\begin{tabular}{|c|c|c|c|c|c|c|c|}
\hline \multirow[b]{3}{*}{ MAP Rate } & \multirow[b]{3}{*}{ Yield } & \multirow[b]{3}{*}{$\mathrm{N}$ uptake } & \multicolumn{3}{|c|}{ Protein } & \multirow{2}{*}{\multicolumn{2}{|c|}{ Starch }} \\
\hline & & & \multicolumn{2}{|c|}{ Novelty } & \multirow[b]{2}{*}{ Albany ${ }^{\dagger}$} & & \\
\hline & & & 2010 & 2011 & & Novelty $^{\dagger}$ & Albany $^{\dagger}$ \\
\hline $\mathrm{kg} \mathrm{P}_{2} \mathrm{O}_{5} \mathrm{ha}^{-1}$ & $\mathrm{Mg} \mathrm{ha}^{-1}$ & $\mathrm{~kg} \mathrm{ha}^{-1}$ & \multicolumn{3}{|c|}{------- g kg$^{-1}$} & \multicolumn{2}{|c|}{------ g kg $^{-1}$} \\
\hline 0 & 8.44 & 440.7 & 77 & 88 & 85 & 737 & 723 \\
\hline 56 & 8.08 & 429.6 & 77 & 91 & 84 & 735 & 722 \\
\hline 112 & 8.14 & 428.7 & 76 & 91 & 84 & 737 & 723 \\
\hline $\operatorname{LSD}(P=0.1)$ & 0.25 & $\mathrm{NS}^{\ddagger}$ & NS & 2 & NS & 2 & NS \\
\hline
\end{tabular}

'Data were combined over years (2010 and 2011).

${ }^{\mathrm{N} S}=$ Not significant.

At Novelty in 2011, grain protein concentration increased $3 \mathrm{~g} \mathrm{~kg}^{-1}$ with MAP at 56 and $112 \mathrm{~kg} \mathrm{P}_{2} \mathrm{O}_{5} \mathrm{ha}^{-1}$ compared to the non-treated control, but at Novelty in 2010 and Albany (2010 and 2011) no differences were observed (Table 6). MAP at 0 and $112 \mathrm{~kg} \mathrm{P}_{2} \mathrm{O}_{5} \mathrm{ha}^{-1}$ increased starch concentration $2 \mathrm{~g} \mathrm{~kg}^{-1}$ over MAP at $56 \mathrm{P}_{2} \mathrm{O}_{5}$ $\mathrm{ha}^{-1}$ at Novelty, but not Albany. Plant N, P, K uptake, and APRE were not affected by MAP rate during all four site-years evaluated in this research (data not presented).

\section{Conclusions}

The two $\mathrm{P}$ enhanced efficiency products this study evaluated did not consistently increase agronomic performance, including apparent $P$ recovery efficiency, at the sites and environmental conditions in interaction with several fertilization rates and tillage practices evaluated in this research. Additionally, the $\mathrm{P}$ enhancers did not affect plant population, silage dry weights, grain moisture, yield, grain protein, grain starch, $\mathrm{N}, \mathrm{K}$ uptake, or apparent P recovery efficiency. In the NT-BC and ST-DB treatments, the addition of Avail ${ }^{\circledR}$ or $\mathrm{P}_{2} \mathrm{O}_{5}$-Max ${ }^{\circledR}$ did not increase $\mathrm{P}$ uptake over non-treated controls. Since soils in this study were acidic, more research should be performed evaluating Avail ${ }^{\circledR}$ or $\mathrm{P}_{2} \mathrm{O}_{5}-\mathrm{Max}^{\circledR}$ use on alkaline soils. Strip-till/deep banding increased plant populations by 3,500 to 15,500 plants $\mathrm{ha}^{-1}$ compared to NT/broacast. At Novelty, greater plant populations associated with ST increased grain yields $1.57 \mathrm{Mg} \mathrm{ha}^{-1}$ compared to NT. Poorly drained claypan soils in Northeast Missouri responded more to ST than silty clay soils in Northwest Missouri. Corn grain yields with MAP at $0 \mathrm{~kg} \mathrm{ha}^{-1}$ were 0.30 to $0.36 \mathrm{Mg} \mathrm{ha}^{-1}$ more than MAP at 56 or $112 \mathrm{~kg} \mathrm{P}_{2} \mathrm{O}_{5} \mathrm{ha}^{-1}$. This difference may be due to adding ammonium nitrate to balance the $\mathrm{N}$ contribution as the MAP rate increased to $112 \mathrm{~kg} \mathrm{ha}^{-1}$.

\section{References}

Al-Kaisi, M., \& Kwaw-Mensah, D. (2007). Effect of tillage and nitrogen rate on corn yield and nitrogen phosphorus uptake in a corn-soybean rotation. Agronomy J., 99, 1548-1558. http://dx.doi.org/10.2134/agronj2007.0012

Al-Kaisi, M., \& Licht, M. A. (2004). Effect of strip tillage on corn nitrogen uptake and residual soil nitrate accumulation compared with no-tillage and chisel plow. Agronomy J., 96, 1164-1171. http://dx.doi.org/10.2134/agronj2004.1164

Archer, D. W., \& Reicosky, D. C. (2009). Economic performance of alternative tillage systems in the northern corn belt. Agronomy J., 101, 296-304. http://dx.doi.org/10.2134/agronj2008.0090x

Bauder, J. W., Randall, G. W., \& Swann, J. B. (1981). Effect of four continuous tillage systems on mechanical impedance of a clay loam soil. Soil Sci. Soc. Am. J., 45, 802-806. http://dx.doi.org/10.2136/sssaj1981.03615995004500040026x

Bolton, F. E., \& Booster, D. E. (1981). Strip-till planting in dryland cereal production. Trans. ASAE, 24, 59-62. 
Borges, R., \& Mallarino, A. P. (2001). Deep banding phosphorus and potassium fertilizers for corn managed with ridge tillage. Soil Sci. Soc. Am. J., 65, 376-384. http://dx.doi.org/10.2136/sssaj2001.652376x

Burrows, W. C., \& Larson, W. E. (1962). Effect of amount of mulch on soil temperature and early growth of corn. Agronomy J. 54, 19-23. http://dx.doi.org/10.2134/agronj1962.00021962005400010007x

Dick, W. A., \& Van Doren, Jr., D. M. (1985). Continuous tillage and rotation combinations effects on corn, $\begin{array}{llllll}\text { soybean, and } \quad \text { oat } & \text { yields. }\end{array}$ http://dx.doi.org/10.2134/agronj1985.00021962007700030023x

Drury, C. F., Tan, C. S., Reynolds, W. D., Welacky, T. W., Weaver, S. E., Hamill, A. S., \& Vyn, T. J. (2003). Impacts of zone tillage and red clover on corn performance and soil physical quality. Soil Sci. Soc. Am. J., 67, 867-877. http://dx.doi.org/10.2136/sssaj2003.8670

Erbach, D. C., Benjamin, J. G., Cruse, R. M., Elamin, M. A., Mukhtur, S., \& Choi, C. H. (1992). Soil and corn response to tillage with paraplow. Trans. ASAE, 35, 1347-1354.

Fortin, M. C., \& Pierce, F. J. (1990). Developmental and growth effects of crop residues on corn. Agronomy J., 82, 710-715. http://dx.doi.org/10.2134/agronj1990.00021962008200040013x

Griffith, D. R., Mannering, J. V., Galloway, H. M., Parson, S. D., \& Rickey, C. B. (1973). Effect of eight tillage planting systems on soil temperature, percent stand, plant growth, and yield of corn on five Indiana soils. Agronomy J., 65, 321-326. http://dx.doi.org/10.2134/agronj1973.00021962006500020040x

Griffith, D. R., \& Wollenhaupt, N. C. (1994). Crop residue management strategies for the midwest. In J. L. Hatfield, \& B. A. Steward (Eds.), Crop residue management (pp. 15-37). Lewis Publ., Boca Raton, FL.

Guy, S. O., \& Oplinger, E. S. (1989). Soybean cultivar performance as influenced by tillage system and seed treatment. J. Prod. Agric., 2, 57-62.

Hairston, J. E., Jones, W. F., McConnaughey, P. K., Marshall, L. K., \& Gill, K. B. (1990). Tillage and fertilizer management effects on soybean growth and yield on three Mississippi USA soils. J. Prod. Agric., 3, 317-323.

Halvorson, A. D., Mosier, A. R., Reule, C. A., \& Bausch, W. C. (2006). Nitrogen and tillage effects on irrigated continuous corn yields. Agronomy J., 98, 63-71. http://dx.doi.org/10.2134/agronj2005.0174

Hill, R. L. (1990). Long-term conventional and no-tillage effects on selected soil physical properties. Soil Sci. Soc. Am. J., 54, 161-166. http://dx.doi.org/10.2136/sssaj1990.03615995005400010025x

Howard, D. D., Essington, M. E., \& Logan, J. (2002). Long-term broadcast and banded phosphorus fertilization of corn produced using two tillage systems. Agronomy J., 94, 51-56. http://dx.doi.org/10.2134/agronj2002.5100

Hussain, I., Olson, K. R., \& Ebelhar, S. A. (1999). Impacts of tillage and no-till on production of maize and soybean on an eroded Illinois silt loam soil. Soil Tillage Res., 52, 37-49.

Licht, M. A., \& Al-Kaisi, M. (2005a). Corn response, nitrogen uptake, and water use in strip-tillage compared with no-tillage and chisel plow. Agron. J., 97, 705-710. http://dx.doi.org/10.2134/agronj2004.0102

Licht, M. A., \& Al-Kaisi, M. (2005b). Strip-tillage effect on seedbed soil temperature and other soil physical properties. Soil Till. Res., 80, 233-249.

Lu, D. Q., Chien, S. H., Chien, Henao, J., \& Sompongse, D. (1987). Evluation of short-term efficiency of diammonium phosphate versus urea plus single superphosphate on calcareous soil. Agronomy J., 79, 896-900. http://dx.doi.org/10.2134/agronj1987.00021962007900050028x

Karamanos, R. E., \& Puurveen, D. (2011.) Evaluation of a polymer treatment as enhancer of phosphorus fertilizer efficiency in wheat. Can. J. Soil Sci., 91, 123-125.

Kuehl, R. O. (1994.) Statistical principles of research design and analysis (p. 686).

Belmont, C. A., Malhi, S. S., Zenter, R. P., \& Heier, K. (2001). Banding increases effectiveness of fertilizer P for alfalfa production. Nutrient Cycling in Agroecosystems, 59, 1-11. Duxbury Press.

Mallarino, A. P., Bordoli, J. M., \& Borges, R. (1999). Phosphorus and potassium placement effects on early growth and nutrient uptake of no-till corn and relationships with grain yield. Agronomy J., 91, 37-45. http://dx.doi.org/10.2134/agronj1999.00021962009100010007x 
Nash, P. R., Motavalli, P. P., \& Nelson, K. A. (2012). Nitrous oxide emissions from claypan soils due to nitrogen fertilizer source and tillage/fertilizer placement practices. Soil Sci. Soc. Am. J., 76, 983-993.

Nathan, M., Stecker, J., \& Sun, Y. (2006). Soil testing in Missouri: A guide for conducting soil tests in Missouri. EC923. Missouri Cooperative Extension Service, Univ. of Missouri-Lincoln Univ., Columbia, MO.

Nelson, K. A., Meinhardt, C. G., \& Smoot, R. L. (2010). Wheat (Triticum acestivum L.) cultivar selection affects double-crop and relay-intercrop soybean (Glycine $\max$ L.) response on claypan soils. Intl. J. Agron. http://dx.doi.org/10.1155/2010/543261.

Overstreet, L. F., \& Hoyt, G. D. (2008). Effects of strip tillage and production inputs on soil biology across spatial gradient. Soil Sci. Soc. Am. J., 72, 1454-1463. http://dx.doi.org/10.2136/sssaj2007.0143

Patrick Jr., W. H., Sloane, L. W., \& Phillips, S. A. (1959). Response of cotton and corn to deep placement of fertilizers and deep tillage. Soil Sci. Soc. Am. J., 23, 307-310. http://dx.doi.org/10.2136/sssaj1959.03615995002300040024x

Perez-Bidegain, M., Cruse, R. M., \& Ciha, A. (2007). Tillage system by planting date interaction effects on corn and soybean yield. Agronomy J., 99, 630-636. http://dx.doi.org/10.2134/agronj2006.0058

Pierce, F. J., Fortin, M. C., \& Staton, M. J. (1992). Immediate and residual effects of zone-tillage in rotation with no-tillage on soil physical properties and corn performance. Soil Tillage Res., 24, 149-164.

Randall, G. W., Vetsh, J. A., \& Murrel, T. S. (2001). Corn response of phosphorus placement under various tillage practices. Better Crops, 85, 12-15.

Robertson, W. K., Hutton, C. E., \& Thompson, L. G. (1958). Response of corn in superphosphate placement

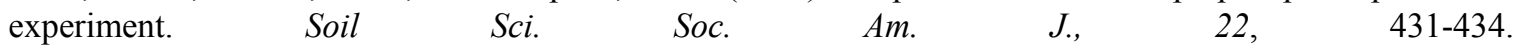
http://dx.doi.org/10.2136/sssaj1958.03615995002200050017x

Rosen's Inc. (2012). P2O5-Max phosphate fertilizer additive for dry fertilizer impregnation. Retrieved from http://fs1.agrian.com/pdfs/P_Max_Label2.pdf

SFP. (2009). Science behind Avail ${ }^{\circledR}$. Specialty Fertilizer Products. Retrieved from http://www.chooseavail.com/Science.aspx

Torbert, H. A., Potter, K. N., Morrison, P., \& Jr., J. E. (2001). Tillage system, fertilizer nitrogen rate, and timing effect on corn yields in the Texas Blackland Prairie. Agronomy J., 93, 1119-1124. http://dx.doi.org/10.2134/agronj2001.9351119x

Triplett, G. B., \& Dick, W. A. (2008). No-Tillage crop production: a revolution in agriculture! Agronomy J., 100, 153-165. http://dx.doi.org/10.2134/agronj2007.0005c

Uri, N. D. (2000). Perceptions of the use of no-till farming in production agriculture in the United States: an analysis of survey results. Agric. Ecosyst. Environ., 77, 263-266. http://dx.doi.org/10.1016/S0167-8809(99)00085-7

USDA. (2011). United States Department of Agriculture National Agriculture Statistics Service: Corn-Quick Stats. $\quad$ Retrieved from http://quickstats.nass.usda.gov/results/FF6C5AFF-A0B0-3ADC-9962-311B64606F83\#6BC4656D-E986-3 103-93A5-EAAF8EFDEBCD

USDA. (2012). United States Department of Agriculture Economic Research Service: Fertilizer Consumption and Use-By Year Table 1. Retrieved from http://www.ers.usda.gov/Data/FertilizerUse/

Vetsch, J. A., \& Randall, G. W. (2002). Corn production as affected by tillage system and starter fertilizer. Agronomy J., 94, 532-540.

Vetsch, J. A., \& Randall, G. W. (2004). Corn production as affected by nitrogen application timing and tillage. Agronomy J., 96, 502-509. http://dx.doi.org/10.2134/agronj2002.5320

Vetsch, J. A., Randall, G. W., \& Lamb, J. A. (2007). Corn and soybean production as affected by tillage systems. Agronomy J., 99, 952-959. http://dx.doi.org/10.2134/agronj2004.5020

Vyn, T. J., \& Raimbult, B. A. (1993). Long-term effect of five tillage systems on corn response and soil structure. Agronomy J., 85, 1074-1079. http://dx.doi.org/10.2134/agronj1993.00021962008500050022x

Ward, N. C. (2010). Impact of Avail and Jumpstart on yield and phosphorus response of corn and winter wheat in Kansas. M. S. thesis, Kansas State Department of Agronomy, College of Agriculture, Kansas State University. 